\title{
Intravenous Leiomyomatosis Extending from the Uterus to the Heart
}

\author{
Mubarak Mohd Yusof', Kama Azira Awang Ramli', \\ Zainab Yahaya², Abd Kahar Ghapar ${ }^{3}$, Noordini Mohamed Dani4, \\ Hamidah Ismail ${ }^{4}$, Mohamad Arif Muhammad Nor ${ }^{5}$, \\ Hamdan Leman ${ }^{5}$, Yusri Mohammed ${ }^{1}$ \\ 'Departments of Radiology, ${ }^{2}$ Obstetrics and Gynecology, ${ }^{3}$ Cardiology, ${ }^{4} \mathrm{Cardiac}$ Anesthesiology, \\ ${ }^{5}$ Cardiothoracic Surgery, Hospital Serdang, Selangor, Malaysia
}

Received: November 18, 2018

Revised: April 12, 2019

Accepted: April 18, 2019

Corresponding author

Mubarak Mohd Yusof, MD, Mrad

Department of Radiology,

Hospital Serdang, Jalan Puchong,

43000 Kajang, Selangor, Malaysia

Tel: 60-1-3622-2369

Fax: 60-3-8947-5322

E-mail: adibawazif@yahoo.com

\begin{abstract}
We report a case of intravenous leiomyomatosis with right atrium extension through the left gonadal vein, left renal vein and inferior vena cava. The tumor moved in and out of the right ventricle via the tricuspid valve, which could lead to valve obstruction and sudden death. We illustrate the importance of multimodality cardiac imaging to diagnose this condition by performing echocardiography, cardiac magnetic resonance imaging and computed tomography.
\end{abstract}

Key words Inferior vena cava · Tricuspid valve - Cardiac tumor - Uterine fibroid · CT angiography.

\section{INTRODUCTION}

Intravenous leiomyomatosis (IVL) is a benign smooth muscle cell tumor of uterine origin. The tumor can extend through the pelvic veins, renal veins and inferior vena cava (IVC) into the right atrium (RA) or as far as the pulmonary artery [1]. IVL can cause dyspnea, chest pain, heart failure and sudden death $[2,3]$.

\section{CASE REPORT}

A 52-year-old woman with four children, with the last childbirth occurring 14 years ago presented with menorrhagia for the past three months. She also had abdominal discomfort one month prior to the current admission. Her body weight was significantly reduced by $20 \mathrm{~kg}$ in three months. During clinical examination, her blood pressure was 118/90 $\mathrm{mm} \mathrm{Hg}$ and a series of pulse rate measurements showed tachycardia that ranged from $116 / \mathrm{min}$ to $120 / \mathrm{min}$. There was a mass in the mid-lower abdomen that measured $5 \times 8 \mathrm{~cm}$. No other abnormalities were found.

The patient underwent ultrasound (US) of the abdomen which showed a lobulated left adnexal mass that measured $11 \times 7$ $\mathrm{cm}$. Echocardiography (ECHO) and thyroid function test were

(a) This is an Open Access article distributed under the terms of the Creative Commons Attribution Non-Commercial License (https://creativecommons.org/licenses/by$\mathrm{nc} / 4.0$ ) which permits unrestricted non-commercial use, distribution, and reproduction in any medium, provided the original work is properly cited. performed in view of the tachycardia. There was an elongated mass in the RA that moved in and out of the right ventricle (RV) through the tricuspid valve (Fig. 1). The ejection fraction was $77 \%$ and no regional wall motion abnormalities were noted. All chambers were normal in size with mild tricuspid regurgitation.

The patient then underwent a computed tomography (CT) scan of the thorax and abdomen. The CT scan revealed a welldefined soft tissue mass in the RA that extended into the IVC. The mass did not attach to the wall of the IVC. This mass further extended into the left renal and left gonadal veins. The Hounsfield unit of the mass was from 58 to 74 . There was also a lobulated, enhancing soft tissue mass with a density unit from 40 to 68 in the left adnexa, as depicted in the abdominal US. This mass measured $12.2 \times 7.9 \mathrm{~cm}$ and originated from the uterus, and the uterus was noted to be bulky. There was no clear margin between the adnexal mass and the left gonadal vein (Fig. 1).

The patient later underwent cardiac magnetic resonance imaging (MRI) to exclude RA thrombus, and MRI of the abdomen for further evaluation of the adnexal mass. The mass in the RA measured $6.5 \times 2.4 \mathrm{~cm}$ and was not attached to the RA wall and septum. The mass moved in and out of the RV via the tricuspid valve, as seen in the ECHO. The mass was isointense to the myocardium on $\mathrm{T} 1$ and $\mathrm{T} 2$ weighted images, and was enhanced after gadolinium (Fig. 2). The left adnexal mass and the mass in the left gonadal vein were also enhanced. From the imaging mo- 

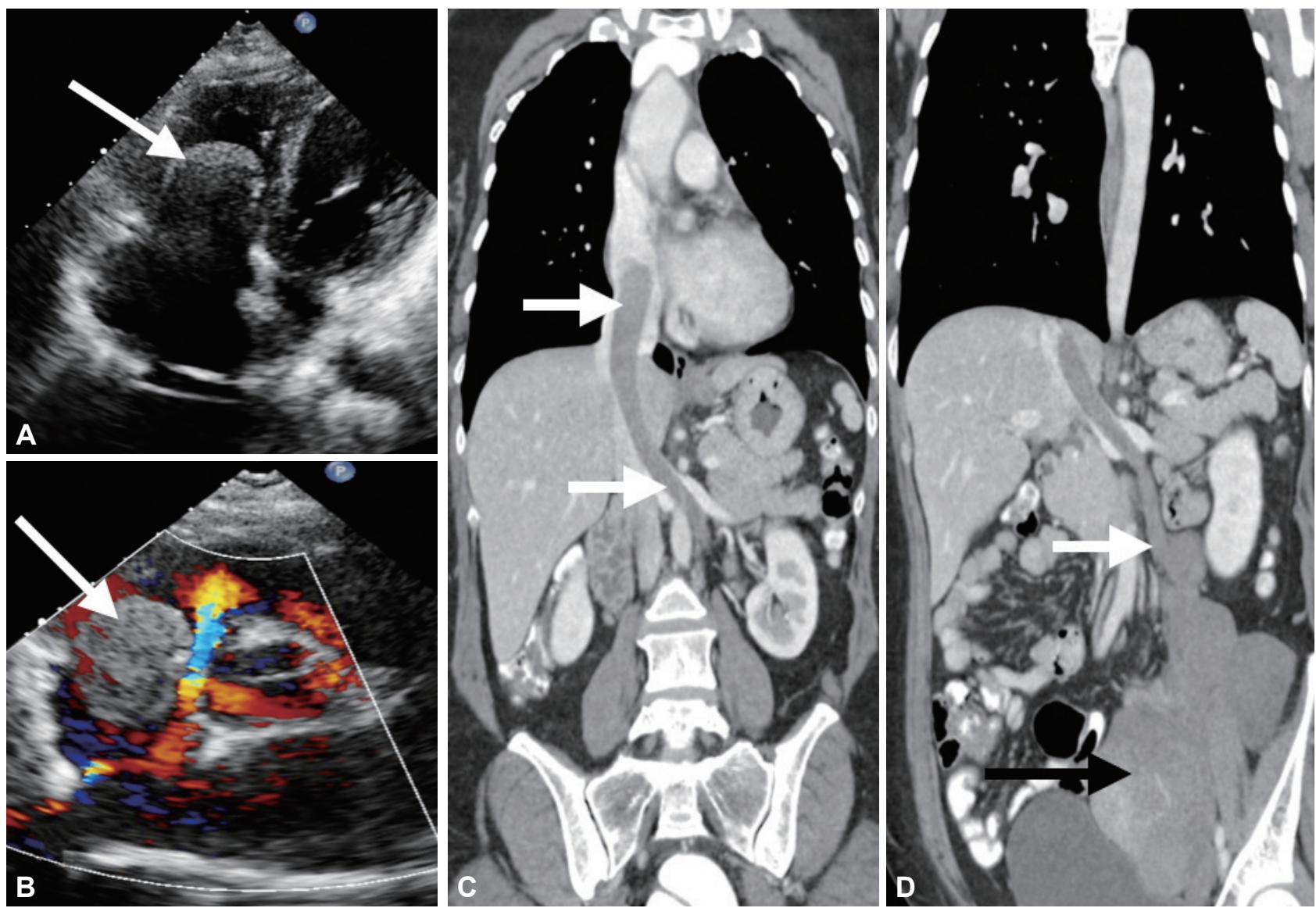

Fig. 1. A 52-year-old woman with intravenous leiomyoma extending into the right atrium. (A and B) Echocardiography images showing the echogenic mass (white arrows) protruding from the right atrium to the right ventricle with minimal Doppler signal within the mass. (C and D) Coronal computed tomography reconstruction images revealed a relatively hypodense mass in the right atrium, inferior vena cava and left gonadal vein (white arrows) and in continuation with the lobulated adnexal mass (black arrow).

dalities, the mass was suggestive of IVL with extension into the RA through the left gonadal vein, left renal vein and IVC.

A specimen of the abdominal mass was obtained by a transabdominal biopsy, guided by US. The results revealed a fibrofatty tissue with no evidence of inflammation, thrombosis, hemorrhage, mitosis, and malignancy. The patient later underwent two stages of surgery. The first surgery was to remove the mass in the RA and IVC (Fig. 3). After 3 months, she had a total abdominal hysterectomy with a bilateral salpingo-oophorectomy and removal of the left adnexal mass. Both surgeries were uneventful and she is currently under follow-up for her hyperthyroidism. The histopathology of the RA mass was atypical leiomyomatosis. The mass in the left adnexa was diagnosed as IVL and leiomyoma of the uterus.

\section{DISCUSSION}

An IVL is a rare [1] and potentially life-threatening tumor [2]. It was first described by Birch-Hirschfeld in 1896 [3] while IVL with the cardiac extension was first reported in 1907 by Dürck
$[2,4]$. An extrauterine leiomyoma occurs in $30 \%$ of cases and IVL with the cardiac extension has been reported in approximately $10 \%$ of cases [1]. In 2015, Fornaris et al. [2] reported a case of IVL with extension to the pulmonary artery.

Two theories about the origin of IVL have been proposed [25]. The first theory, the Knauer theory, suggested that the tumor originated from the smooth muscle of the vascular wall. The second theory, the Sitzenfry theory, suggested that the tumor was from the smooth muscle of a uterine leiomyoma that invaded the blood vessel [5]. In this case, the tumor was slow growing and extended into the vessel lumen without invasion [6]. Based on reports, IVL extension is common through the lumen of the iliac vein to the IVC; however, in certain cases, the ovarian vein was the alternative route of extension [6]. In this case, the IVL was from the uterine leiomyoma with extension to the right heart chamber via the left gonadal vein.

IVL clinical manifestations are nonspecific and most patients are asymptomatic $[3,4]$. Patients could present with abdominal pain or a mass caused by the uterine leiomyoma. Cases of IVL and intracardiac extension with the previous history of hyster- 

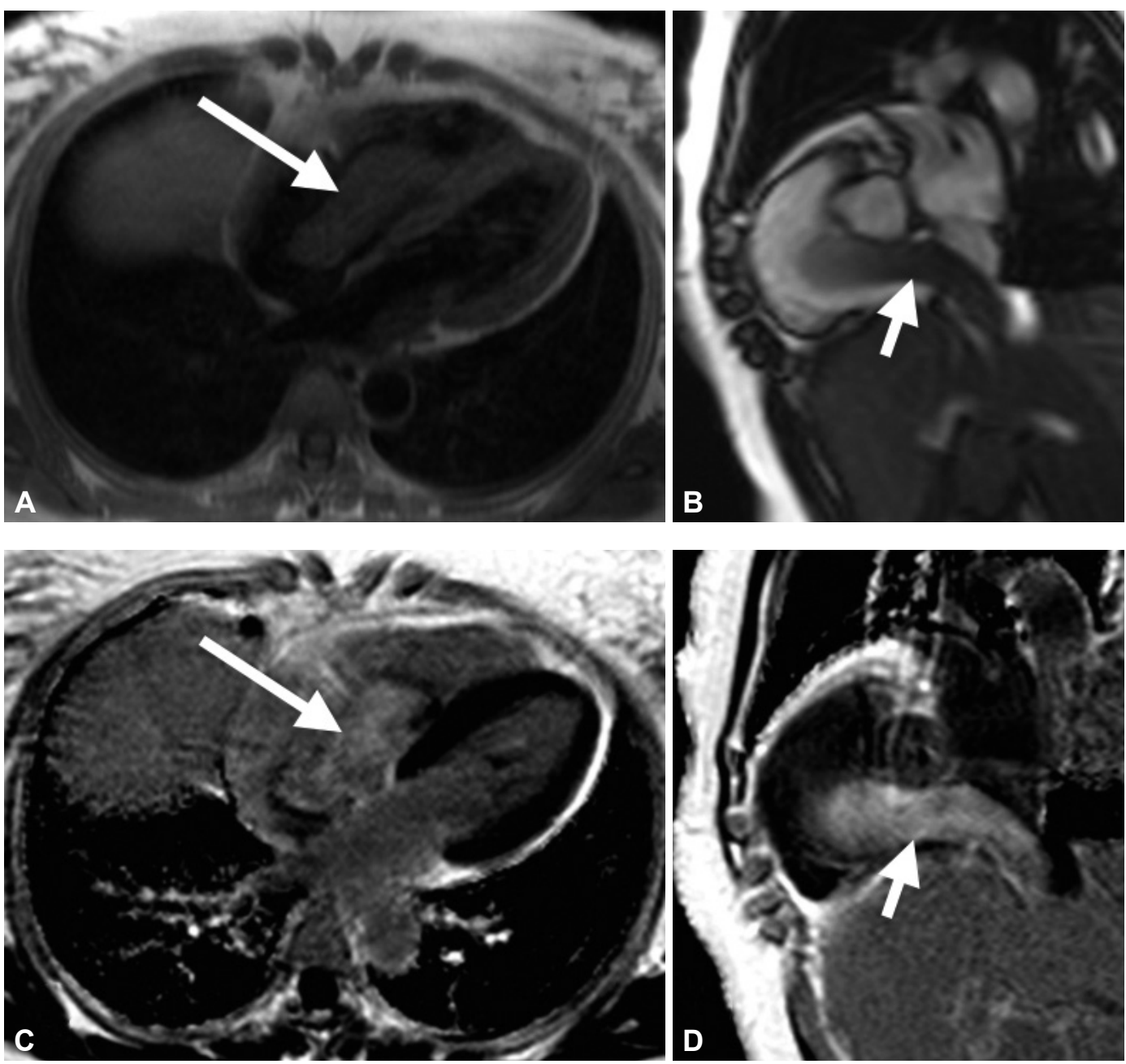

Fig. 2. A 52-year-old woman with IVL extending into the right atrium. (A-D) MRI images showing the mass (long arrow) is isointense to myocardium on the T1 Turbo Spin-Echo 4 chamber view (A) and enhances (long arrow) in T1 Phase Sensitive Inversion Recovery 4 chamber view (C). Static frame of a cine Steady State Free Precession aortic valve view (B) showing the mass (short arrow) in the distal IVC and right atrium and the mass enhances (short arrow) in T1 Phase Sensitive Inversion Recovery aortic valve view (D).

ectomy have also been reported $[1,6]$. Therefore, symptoms such as dyspnea and chest pain or symptoms related to IVC obstruction could be related to an intracardiac mass [3,5]. In this reported case, the RA mass had life-threatening potential, because it could have caused tricuspid valve obstruction and sudden death [4].

On ECHO, IVL with cardiac extension might be seen as an elongated hyperechoic mobile mass from the IVC into the RA. The mass could occasionally protrude in and out of the RV during the cardiac cycle [2]. A CT scan with imaging reconstruction showed the uterine leiomyoma extension into the pelvic vein, renal vein, IVC and RA. The mass caused a filling defect which was relatively hypodense to the contrast in the affected vessels [2,7]. Cardiac MRI is advantageous for differentiating IVL with bland thrombus. The IVL showed heterogeneous enhancement while the bland thrombus could remain hypointense [3]. Finally, atrial myxoma usually has a narrow base attached to

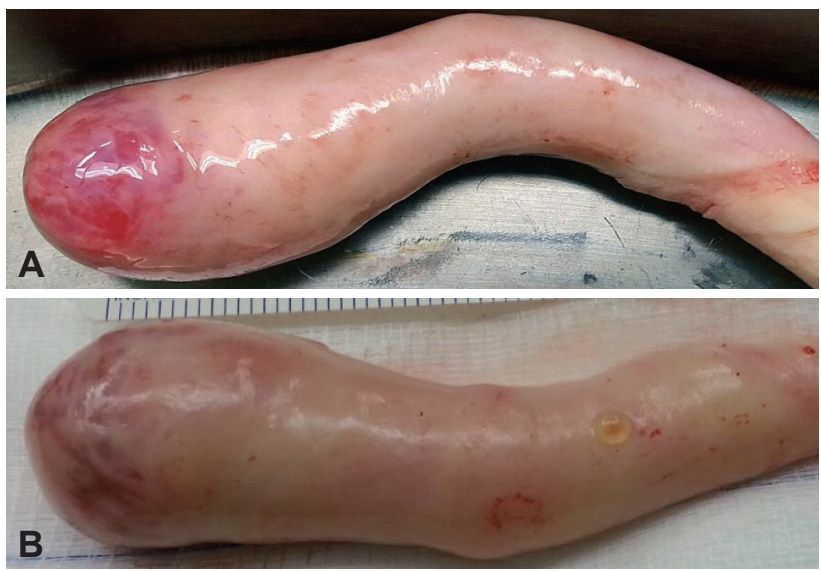

Fig. 3. A 52-year-old woman with intravenous leiomyomatosis extending into the right atrium. (A and B) Gross specimen of the resected mass in the right atrium and inferior vena cava. The mass consisted of a whitish and firm polypoidal tissue that weighed $37.5 \mathrm{~g}$. The cut section of the mass shows multiple cystic areas, especially at the surface of the mass. 
the interatrial septum and does not involve the IVC [3].

In this case and in similar cases, surgery was mandatory for patients IVL $[4,5]$. Surgery was performed to completely remove the uterine leiomyoma and IVL extension in the veins and RA. Complete removal of the entire tumor was mandatory as the recurrence rate was about $30 \%$ [5].

\section{Conflicts of Interest}

The authors declare that they have no conflict of interest.

\section{REFERENCES}

1. Wang HC, Wang YB, Chen XH, Cui LL. Uterine intravenous leiomyomatosis with intracardiac extension and pulmonary benign metastases on FDG PET/CT: a case report. Korean J Radiol 2016;17:289-294.

2. Fornaris RJ, Rivera M, Jiménez L, Maldonado J. Multimodality evaluation of intravenous leiomyomatosis: a rare, benign but potentially life-threatening tumor. Am J Case Rep 2015;16:794-800.

3. Gunderson CC, Parsons B, Penaroza S, Peyton MD, Landrum LM. Intravenous leiomyomatosis disguised as a large deep vein thrombosis. J Radiol Case Rep 2016;10:29-35.

4. Gui T, Qian Q, Cao D, Yang J, Peng P, Shen K. Computerized tomography angiography in preoperative assessment of intravenous leiomyomatosis extending to inferior vena cava and heart. BMC Cancer 2016;16:73.

5. Zhang G, Yu X, Lang J. Intravenous leiomyomatosis with inferior vena cava or intracardiac extension and concurrent bilateral multiple pulmonary nodules: a report of 2 cases. Medicine (Baltimore) 2016;95:e4722.

6. Deac MO, Sheppard MN, Moat N, Burke SJ, Christmas T, Mohiaddin RH. Images in cardiovascular medicine. From uterus to pulmonary embolus: an uncommon association. Circulation 2009;120:e16-e19.

7. Ouyang A, Su X, Li S, Li K, Wang J. Imaging manifestations of intravascular leiomyoma involving the right atrium: a case report and literature review. Int J Clin Exp Med 2015;8:11602-11606. 